Annuaire suisse de politique de développement

27-2 | 2008

Migration et développement: un mariage arrangé

\title{
La contribution des migrants au développement local en Amérique latine
}

Claude Auroi

\section{OpenEdition}

1 Journals

Édition électronique

URL : http://journals.openedition.org/aspd/195

DOI : 10.4000/aspd.195

ISSN : 1663-9669

Éditeur

Institut de hautes études internationales et du développement

Édition imprimée

Date de publication : 1 décembre 2008

Pagination : 133-153

ISBN : 978-2-940415-07-6

ISSN : 1660-5934

\section{Référence électronique}

Claude Auroi, «La contribution des migrants au développement local en Amérique latine », Annuaire suisse de politique de développement [En ligne], 27-2 | 2008, mis en ligne le 22 mars 2010, consulté le 07 septembre 2020. URL : http://journals.openedition.org/aspd/195 ; DOI : https://doi.org/10.4000/ aspd. 195

(c) The Graduate Institute I Geneva 


\title{
La contribution des migrants au développement local en Amérique latine
}

\author{
Claude Auroi*
}

\section{Introduction}

Depuis une dizaine d'années, les migrations mondiales ont pris une importance croissante. Ces migrations, dans un contexte de globalisation, concernent maintenant presque toutes les régions de la planète. En ce qui concerne l'Amérique latine, le nombre des migrants vers les Etats-Unis et d'autres pays du continent et vers l'Europe a plus que doublé entre 1990 et 2000.

Un des aspects socio-économiques les plus significatifs alliés au mouvement de la migration est celui des flux d'argent et de produits que les migrants envoient à leurs familles. Du fait de l'augmentation de la migration, le volume des transferts de fonds des migrants d'Amérique latine a lui aussi énormément augmenté, de $15 \%$ par an depuis une vingtaine d'années ${ }^{1}$. De 5,8 milliards de dollars en 1990, ces transferts ont atteint officiellement 42,4 milliards de dollars en $2005^{2}$.

A partir du début des années 2000, les organismes financiers internationaux (Fonds monétaire international, Banque mondiale, Banque interaméricaine de développement) et les organisations spécialisées (Organisation internationale pour les migrations, Programme des Nations unies pour le développement) se sont penchés sur le phénomène des transferts de fonds et ses conséquences aux niveaux macro- et microéconomique. Les études globales ${ }^{3}$ dont nous disposons aujourd'hui permettent de se faire une idée relativement précise des effets des transferts sur un certain nombre de paramètres économiques et sociaux.

Dans cet article, ce sera surtout la relation entre les transferts de fonds des migrants et le développement économique local et régional en Amérique latine

* Professeur à l'Institut de hautes études internationales et du développement, Genève.

1 Inter-American Development Bank (IDB), Remittance Flows to Latin America and the Caribbean, 2004, Washington, DC, IDB, 2004. World Bank, Global Economic Prospects 2006: Economic Implications of Remittances and Migration, Press launch, with Christopher Neal, François Bourguignon, Hans Timmer, Dilip Ratha, William Shaw, Washington DC, World Bank, 16 November 2005.

2 Bimal Gosh, Migrants' Remittances and Development: Myths, Rhetoric and Realities, Geneva, International Organization for Migration (IOM), 2006, p. 12, tableau 1.

3 Voir les études suivantes de la Banque américaine de développement (référencées plus complètement dans la bibliographie à la fin de cet article): Survey of Remittance Senders: United States to Latin America (2002), Remittance Senders and Receivers: Tracking the Transnational Channels (2003), Sending Money Home: Remittance Recipients in the Dominican Republic and Remittance Senders from the United States (2004), State by State Survey of Remittance Senders: United States to Latin America (2004), Remittance Flows to Latin America and the Caribbean (2004, op. cit.), Remittances 2005: Promoting Financial Democracy (2006), Enviando Dinero a Casa: Marcador de la Industria de Remesas (2006); ainsi que: Pablo Fajnzylber and J. Humberto López, Close to Home: The Development Impact of Remittances in Latin America, Conference Edition, Washington, DC, World Bank, 2007. 
qui sera abordée. Dans un premier temps, nous montrerons quels sont les effets macroéconomiques pour les pays recevant les transferts. Cependant, comme le phénomène des transferts de fonds a un aspect fondamentalement «micro » en ce qu'il concerne des fonds privés dans un cadre généralement familial, nous verrons ensuite quel usage les receveurs font de leur argent. Il est avéré par de nombreuses études qu'entre $75 \%$ et $80 \%$ des dépenses des familles de migrants sont destinées à l'alimentation, l'habillement, le confort ménager et la santé. Si l'on y ajoute les dépenses pour l'éducation, ce ne sont guère que $5 \%$ à $10 \%$ qui sont destinés à des dépenses que l'on peut considérer comme de l'investissement, tels l'achat de terres ou de biens immobiliers ou l'ouverture de petits commerces et entreprises. C'est pourquoi il a été souvent dit que les transferts de fonds sont essentiellement un moyen de lutter contre la pauvreté - de permettre la survie des membres des familles restés au pays.

Mais certaines études ont aussi catégorisé le terme «investissement » de manière plus détaillée, faisant entrer dans cette catégorie les biens durables et l'éducation $^{4}$. Sur cette base, nous nous demanderons si les stratégies de dépense choisies par les familles transnationales de migrants sont cohérentes par rapport à leur avenir.

C'est dans le cadre plus restreint des communautés et des régions de forte émigration que se trouve sans doute une des clés de compréhension des changements que la migration entraîne. Les changements au niveau rural retiendront plus particulièrement notre attention, car les effets de l'accroissement des fonds se font sentir sur l'intensité de l'exploitation des terres, les modifications des plans de culture, l'achat de terrains, la disponibilité en main-d'œuvre, le travail féminin et l'émigration rurale vers les villes.

La migration et les fonds envoyés par les migrants ont sans doute aussi une forte influence sur les structures sociopolitiques et de la gouvernance communale; dans les communautés à forte prédominance indigène, ils modifient la relation entre groupes sociaux et groupes ethniques.

$\mathrm{Au}$ vu de la diversité du sous-continent, il serait exagéré de vouloir extrapoler des tendances à toute l'Amérique latine à partir de cas particuliers, même nationaux. Chaque pays, chaque région a ses spécificités quant au contexte économique, institutionnel et financier, par rapport au type de migration et de migrants, aux modes de transfert de fonds et aux possibilités d'investissement. Il n'en reste pas moins vrai qu'il existe aussi des «fondamentaux» de la migration et des transferts en argent et en nature, qui pour l'Amérique latine sont la prédominance de la migration vers les Etats-Unis (75\%) et l'Espagne, l'envoi régulier de fonds sur de nombreuses années et des schémas quasiment stéréotypés de la dépense et des modes d'accumulation (investissement) - lorsque celle-ci peut se réaliser.

Si les effets de la migration vont dans le sens d'un changement social que l'on peut qualifier de positif en ce qu'il stimule l'ouverture nationale et internationale et l'esprit d'initiative, d'autres effets s'avèrent perturbateurs, voire indésirables, qui sont des «effets secondaires» des changements socio-économiques. Ces questions touchent à l'intensité du travail et à la participation au monde du

4 Pablo Fajnzylber and J. Humberto López, op. cit. Jim Airola, «The Use of Remittance Income in Mexico (Report)», International Migration Review, vol. 41, nº 4, 2007. 
travail, aux fluctuations des prix des biens et services, aux relations de genre dans les familles de migrants, à l'éducation des enfants, à l'utilisation dispendieuse ou de prestige de l'argent pour renforcer le capital social et aux choix dans l'investissement.

La perspective d'analyse que nous retenons est celle d'un développement durable pour les familles de migrants pauvres et leurs communautés. Les familles font-elles de bons choix par rapport à leurs capacités de survie pendant et après la migration? Les relations entre migrants et non-migrants peuventelles faire progresser toute la communauté ou mènent-elles à une augmentation des inégalités sociales, voire à la marginalisation de certains groupes ?

Des réponses exhaustives à toutes les facettes de ces questions sont pour le moment impossibles sur la base des études de cas dont nous disposons pour la période récente et compte tenu de la dimension de cette contribution. Nous nous fondons ainsi essentiellement sur deux types de sources: d'une part, les études et rapports de synthèse d'études antérieures (par exemple celles de la Banque interaméricaine de développement ou de la Banque mondiale), d'autre part, les études résumant un certain nombre de cas pratiques, notamment pour le Mexique ${ }^{5}$, ainsi que quelques études plus récentes de communautés, pour Saint-Domingue, la Bolivie, l'Equateur, le Pérou et le Mexique. Mais il serait présomptueux de prétendre qu'il s'agit ici d'un article de synthèse de toute la littérature sur le sujet, ou d'affirmer que nous disposons d'études de terrain, de thèses et de mémoires en suffisance pour procéder à des comparaisons exhaustives.

\section{Le volumes des flux et l'importance économique globale}

En 2005, les transferts de fonds des migrants vers 1'Amérique latine ont atteint officiellement un niveau de plus de 50 milliards de dollars ${ }^{6}$, auxquels il faut ajouter les sommes qui ne sont pas recensées par les banques ou agences de transfert et qui ont probablement atteint $50 \%$ du total ci-dessus. Cet apport revêt évidemment une grande importance pour les économies des pays concernés. Il est supérieur au montant des exportations et aussi à l'ensemble des aides internationales et des investissements directs. Il permet par conséquent d'atténuer considérablement le fardeau de la dette et rééquilibre la balance des paiements.

Les montants des transferts de fonds peuvent constituer des parts plus ou moins importantes du produit intérieur brut (PIB); elles se situent par exemple à 1,8\%

5 Voir: Jorge Durand and Douglas S. Massey, «Mexican Migration to the US: A Critical Review», Latin American Research Review, vol. 27, n² 2, 1992, pp. 3-43. Fernando Lozano Ascencio, «De excluidos sociales a heroes sexenales. Discurso oficial y remesas en Mexico», in Raúl Delgado Wise y Beatrice Knerr (coord.), Contribuciones al análisis de la migración internacional y el desarrollo regional en México, Universidad Autónoma de Zacatecas, 2005, pp. 41-65.

6 Inter-American Dialogue (IAD), Making the Most of Family Remittances, Second Report of the InterAmerican Dialogue Task Force on Remittances, Washington, DC, IAD, 2007. Les organismes de la Banque interaméricaine de développement (IDB) donnent le chiffre de 42,4 milliards de dollars pour 2005, mais celui-ci ne comprend que les versements officiels passant par la comptabilisation des Banques centrales des Etats. Les transferts de fonds non comptabilisés peuvent être estimés à $50 \%$ du total officiel. Etant donné la marge d'incertitude, une estimation entre 50 et 60 milliards de dollars est raisonnable. 
du PIB au Costa Rica, 2,8\% au Mexique, 34,3\% au Guyana, 20,7\% en Haïti, 9,1\% en République dominicaine, $8,5 \%$ en Bolivie et 3,2\% au Pérou ${ }^{7}$. C'est lorsque l'on compare la valeur des transferts de fonds à la valeur des exportations, et notamment des exportations agricoles, que l'on se rend compte de leur poids dans les différentes économies (tableau 1).

Tableau 1: Comparaison des transferts entre pays

\begin{tabular}{lrrrrrr}
\hline Pays & $\begin{array}{c}\text { Valeur des } \\
\text { versements } \\
\text { (mio dollars) }\end{array}$ & $\begin{array}{c}\text { Population } \\
\text { (mio) }\end{array}$ & $\begin{array}{c}\text { Valeur des } \\
\text { versements } \\
\text { par habitant } \\
\text { (dollars) }\end{array}$ & $\begin{array}{c}\text { Valeur des } \\
\text { versements } \\
\text { en \% du } \\
\text { PIB }\end{array}$ & $\begin{array}{c}\text { Valeur des } \\
\text { versements } \\
\text { en \% des } \\
\text { exportations }\end{array}$ & $\begin{array}{c}\text { Versements } \\
\text { en \% des } \\
\text { exportations } \\
\text { agricoles }\end{array}$ \\
\hline Belize & 81 & 0.27 & 300 & 9 & 23 & 64 \\
\hline Bolivie & 860 & 9.18 & 93.7 & 8.5 & 33 & 68 \\
\hline Colombie & 4126 & 45.6 & 90.5 & 4.1 & 19 & 114 \\
\hline Costa Rica & 362 & 4.33 & 83.6 & 1.8 & 5 & 16 \\
\hline Rép. dominicaine & 2682 & 8.4 & 319.3 & 9.1 & 45 & 377 \\
\hline Equateur & 2005 & 13.23 & 151.5 & 6.4 & 20 & 89 \\
\hline El Salvador & 2830 & 6.88 & 411.3 & 17.1 & 80 & 596 \\
\hline Guatemala & 2993 & 12.6 & 237.5 & 9.3 & 77 & 189 \\
\hline Guyana & 270 & 0.75 & 360 & 34.3 & 49 & 75 \\
\hline Haïtí & 1077 & 8.53 & 126.7 & 20.7 & 224 & 5143 \\
\hline Honduras & 1763 & 7.2 & 244.9 & 21.2 & 69 & 138 \\
\hline Jamaïque & 1651 & 2.65 & 623 & 19 & 104 & 562 \\
\hline Mexique & 20034 & 107.3 & 186.7 & 2.8 & 10 & 168 \\
\hline Nicaragua & 850 & 5.49 & 154.8 & 16.9 & 55 & 158 \\
\hline Pérou & 2495 & 28 & 89.1 & 3.2 & 15 & 120 \\
\hline Venezuela & 272 & 26.75 & 10.2 & 0.2 & 0.5 & 123 \\
\hline
\end{tabular}

Source: Elaboré à partir de Inter-American Development Bank (IDB), Remittances 2005: Promoting Financial Democracy, Washington, DC, IDB, 2006, chiffres 2004

Nous avons fait ailleurs une comparaison entre le montant des envois de fonds dans certains pays et la valeur des exportations des principaux produits de ces Etats $^{8}$. Pour la plupart des pays, le principal «produit» d'exportation est le tourisme (sauf pour Belize et la Bolivie) et le second un produit agricole, un produit minier ou un hydrocarbure. Dans tous les cas, à l'exception de la Bolivie, du Costa Rica, de la République dominicaine et du Venezuela, le total des transferts de fonds dépasse largement la valeur du premier produit exporté. C'est aussi le cas par rapport au deuxième produit le mieux exporté et même, comme le montre la dernière colonne du tableau 1, quant à l'ensemble des exportations agricoles. Mais les transferts de fonds ne sont pas destinés aux mêmes acteurs que dans l'agroexportation ou le tourisme; ils vont en général directement à des familles pauvres, tandis que ce sont de grands consortiums qui bénéficient des recettes d'exportation de matières premières ou du tourisme, dont une partie seulement sera redistribuée sous forme de salaires à des ouvriers agricoles ou des petits paysans. Le reste constitue de grandes masses de capitaux qui peuvent être réinvestis, ce qui n'est pas le cas pour les versements personnels des migrants.

7 World Bank, The Development Impact of Workers' Remittances in Latin America, Report, $\mathrm{n}^{\mathrm{o}} 37026$, Washington, DC, World Bank, 2006.

8 Claude Auroi, «Les envois de fonds pour le développement local. Réflexions à partir de cas latinoaméricains », in Isabel Yépez et Gioconda Herrera (dir.), Nouvelles migrations latino-américaines en Europe, Barcelona, Publicacions i Edicions de la Universitat de Barcelona, pp. 169-194. 
Ces versements sont en effet avant tout un transfert d'une partie des gains d'une «famille transnationale ${ }^{9}$ au reste de cette famille qui réside dans un autre pays que celui où le gain a été réalisé. Ces revenus ne s'amalgament pas à d'autres revenus du même type dans des dépôts bancaires, mais sont dépensés rapidement par les familles. Il est donc essentiel de savoir quels montants sont reçus par ces familles mensuellement et annuellement, et ensuite de distinguer les différentes affectations qui en sont faites. Ainsi pourra-t-on se rendre compte de la mesure dans laquelle ces dépenses vont plutôt dans le sens de la consommation courante ou/et aussi dans celui d'une reproduction élargie.

\section{Les transferts de fonds chez les familles}

Les montants des versements des migrants sont très divers selon les pays, aussi bien quant au volume total que par tête d'habitant. Si le Mexique est globalement le premier pays bénéficiaire, avec officiellement 18 milliards de dollars par an, par tête d'habitant c'est la Jamaïque qui totalise le plus, avec plus de 600 dollars par an. Haïti et le Honduras, quant à eux, sont les pays où les versements constituent le plus fort pourcentage du PIB (plus de 20\%) ${ }^{10}$. En général, la région des Caraïbes et de l'Amérique centrale reçoit les plus hauts pourcentages de transferts, ce qui traduit aussi les plus importants pourcentages de migrants vers les Etats-Unis et accessoirement l'Europe. La fuite des cerveaux contribue largement à ces flux financiers, car un niveau d'éducation plus élevé accroît les possibilités d'obtenir un emploi mieux rémunéré dans le pays d'accueil. «Plus de $80 \%$ des détenteurs d'un baccalauréat de pays comme Haïti, la Jamaïque, la Grenade ou le Guyana vivent à l'étranger, la plupart aux Etats-Unis. ${ }^{11}$ On pourrait presque dire que ces îles et ces petits pays des Caraïbes sont devenus des parties d'un grand tout nord-américain, dont l'ALENA ${ }^{12}$ est le symbole.

Les montants transférés sont moins élevés lorsque l'on va vers le sud du continent, où les taux d'émigration sont aussi plus faibles. Ils sont nuls ou insignifiants au Venezuela, au Chili et en Uruguay, faibles aussi au Surinam et dans toute l'Amérique du Sud, par rapport à l'Amérique centrale. Ce qui ne veut cependant pas dire que des montants faibles au regard du PIB global ou per capita soient à négliger, dans la mesure où il faut savoir à qui sont destinés les versements. Sontce plutôt des familles à revenu moyen ou des familles pauvres qui en bénéficient? Comment se fait la répartition? Dans quelles régions spécifiquement?

D'après plusieurs études, les transferts atteignent en moyenne 200 à 300 dollars plusieurs fois par année, voire chaque mois $^{13}$. Mais ces montants varient selon le

9 Manuel Orozco a utilisé et popularisé ce terme. On trouve aussi l'expression «communauté internationale», particulièrement pertinente lorsqu'il s'agit de villages ou de quartiers de forte migration. Manuel Orozco, Transnational Families: Lives on the Edge, but in Pursuit of Change, 2006, cité par Ezra Rosser, Remittances, College of Law Research Paper, $n^{\circ}$ 40, Washington, DC, American University College of Law, 2008, <http://ssrn.com/abstract=1024177>, p. 11 et note 41.

10 World Bank, The Development Impact of Workers' Remittances in Latin America, op . cit.

11 Pablo Fajnzylber and J. Humberto López, op. cit., p. 11 (NDA: notre traduction).

12 ALENA: Accord de libre-échange nord-américain entre le Canada, les Etats-Unis et le Mexique. CARICOM : Marché commun des Caraïbes. Les exportations et importations des membres de CARICOM ainsi que celles du Mexique se font essentiellement avec les Etats-Unis.

13 World Bank, The Development Impact of Workers' Remittances in Latin America, op. cit. 
pays d'accueil, le type d'emploi et le coût de la vie pour le migrant. En 2004, les envois en République dominicaine s'élevaient à 150 dollars en moyenne, pour 12 à 15 envois annuels ${ }^{14}$.

Pour le migrant générateur de versements de longue durée, ceux-ci représentent environ $10 \%$ de ses gains dans le pays d'accueil, parfois plus s'il n'y réside que temporairement. Cela peut paraître peu, mais les salaires versés aux migrants sont de l'ordre de 1500 à 2000 dollars. Le travailleur doit aussi se loger et, parfois, entretenir un ou plusieurs membres de sa famille sur place. Il lui arrive en outre d'investir dans un logement aux Etats-Unis; il accumule de l'argent en espèces en cas de «coup dur» pour lui et surtout au pays. Les envois en espèces réguliers sont complétés par des envois extraordinaires, par exemple pour la construction de la maison, pour l'école ou les fêtes. Dans le rythme des envois, on remarque en République dominicaine un pic des transferts en novembredécembre et une période creuse en mars-avril, avec une différence de près de $50 \%$.

\section{Les mécanismes de transfert et le contrôle des flux}

Raúl Hernandez-Coss divise les parcours des flux en trois étapes ou miles:

1. Le premier mile concerne les motivations portant le migrant dans le pays d'accueil à envoyer des fonds. Ce point ne sera pas examiné dans cet article.

2. Le mile intermédiaire est celui du transfert proprement dit, soit le déplacement physique ou virtuel des fonds générés par le travail ou d'autres moyens, par des voies diverses, institutionnelles ou informelles.

3. Enfin, le dernier mile concerne l'usage qui est fait des fonds dans le pays d'origine, par le migrant ou sa famille ${ }^{16}$.

Pour notre propos, le dernier mile est le plus important car il concerne directement la consommation ou l'investissement des fonds reçus. Mais le mile intermédiaire doit aussi être considéré dans la mesure où il peut réduire les sommes finales allouées, puisqu'il comprend des dépenses de transfert dont le montant peut être plus ou moins important.

Les organismes financiers internationaux et nationaux et les gouvernements consacrent actuellement la plus grande attention au mile intermédiaire. Tout d'abord, ces flux sont assez mal connus car ils sont souvent informels, le montant de ces derniers étant estimé à la moitié des transferts officiels. Ensuite, les transferts officiels sont peu ou pas taxés, ce qui enlève des redevances à l'Etat. Enfin, les envois font l'objet de coûts de transfert relativement élevés (autour de $10 \%$ ), qui réduisent les avoirs des familles migrantes et incitent au transfert direct de main à main. Les coûts de transfert varient selon le montant envoyé;

14 Bendixen and Associates, Remittances and the Dominican Republic: Survey of Recipients in the Dominican Republic, Survey of Senders in the United States, November 2004, <http://www.iadb.org/ NEWS/docs/SurveysRemittanceFlows_e.ppt>.

15 Leonora Suki, Financial Institutions and the Remittance Market in the Domican Republic, New York, Center on Globalization and Sustainable Development, The Earth Institute at Columbia University, 2004, tableau 3 (source: Banque centrale de la République dominicaine), p. 12.

16 Raúl Hernandez-Coss, Lessons from the US-Mexico Remittance Corridor on Shifting from Informal to Formal Transfer System, World Bank Working Paper, nº 47, Washington, DC, World Bank, 2005. 
ils sont plus élevés pour de petits montants (50 dollars $)^{17}$. Sachant qu'un transfert moyen est de 150 à 300 dollars (plusieurs fois par année, voire chaque mois), un taux entre $8 \%$ et $9 \%$ (incluant les différences de taux de change) est souvent dissuasif et incite à privilégier une voie de transfert non déclarée ${ }^{18}$. Par contre, une plus forte institutionnalisation des transferts, à travers des organismes bancaires, conduira à une rétention plus longue des fonds sur des comptes et par conséquent à l'augmentation de l'épargne et à l'utilisation plus fréquente des fonds transférés pour des investissements significatifs. Mais, pour ce faire, il faudra aussi qu'un nombre beaucoup plus élevé de migrants possèdent un compte bancaire. Actuellement, seuls $25 \%$ environ d'entre eux disposent d'un compte aux deux extrémités de la filière ${ }^{19}$. L'accès à une pleine identité bancaire est restreint par plusieurs facteurs: les régulations étatiques de contrôle des situations illégales et du terrorisme (le Patriot Act des Etats-Unis), le coût des services bancaires, les taux de change, la méfiance et l'illettrisme des déposants face à un système qu'ils comprennent mal, la peur de possibles gels et de la taxation des avoirs. Bien qu'en 2004 les Etats d'Amérique latine suivis par le G-7 la même année - aient émis des directives pour promouvoir et faciliter les transferts institutionnels ${ }^{20}$, il est peu probable que la situation s'améliore rapidement. On peut même estimer qu'elle va s'aggraver du fait des régulations fiscales que les Etats d'Amérique latine vont édicter, à l'instar de la Bolivie qui a voulu taxer ces transferts.

Les études de terrain montrent qu'il existe aussi des coutumes relatives aux transferts de fonds. Les Boliviens qui émigrent temporairement en Argentine, par exemple, préfèrent apporter à leur retour leurs avoirs directement dans leurs poches, avec en outre énormément d'ustensiles et d'appareils pour leurs maisons. S'ils ne rentrent pas, ils remettront l'argent et les biens matériels à des paisanos, des habitants de leur village en qui ils ont confiance. Les envois à travers le système officiel sont rares ${ }^{21}$. Mais dans d'autres cas, plus urbains, les transferts se font surtout par voie bancaire, comme entre Turin, en Italie, et Quillabamba, au sud du Pérou, où les deux tiers des fonds passent par le système bancaire $^{22}$. Des considérations de distance et de fréquence des retours expliquent en grande partie les différences d'attitude entre ces deux cas, mais aussi l'étendue du réseau et des services bancaires offerts, par exemple le crédit.

En ce qui concerne les transferts en nature, la distance peu importante entre l'Argentine et le sud de la Bolivie permet une importation directe d'appareils électroménagers, de téléviseurs et de lecteurs de DVD, plus difficiles à faire parvenir par avion. D'une manière générale, les transferts en nature ne sont pas comptabilisés dans les études, mais il est certain que leur apport est important.

17 Par exemple pour la République dominicaine en 2004, le coût de transfert par MoneyGram, Mateo Express et Pronto Envio était de $15 \%$ pour un envoi de 50 dollars, mais seulement de 8,33\% pour un envoi de 150 dollars, et respectivement de $19,98 \%$ et $6,66 \%$ par Western Union. Leonora Suki, op. cit., tableau 10, p. 24.

18 World Bank, The Development Impact of Workers' Remittances in Latin America, op. cit.

19 Pablo Fajnzylber and J. Humberto López, op. cit., p. 56.

20 Ibid., pp. 50-51.

21 Roxana Dulón, Migración transnacional de bolivianos y bolivianas a la Argentina y su impacto en comunidades de origen, Sucre, Fundación Pasos y Grupo Chorlavi (Fondo Mink’a), 2008, p. 35.

22 Alejandrino S. Loaiza, L. Herrea y César Sotomayor, Las Remesas de los Migrantes Quillabambinos en Italia y sus Implicancias en el Desarrollo Local, Cusco, Proyecto de Desarrollo Corredor PunoCusco y Grupo Chorlavi, 2008, p. 4. 
Dans les trois villages étudiés par Roxana Dulón, les apports directs annuels en nature sont plus importants que ceux en espèces, dont les montants et les possibilités d'utilisation monétaire se trouvent de la sorte amoindris ${ }^{23}$.

\section{L'utilisation des fonds}

Nous allons maintenant examiner quelques-uns des aspects qui touchent à l'utilisation des fonds dans les communautés de départ.

Une première remarque s'impose: les fonds ne vont pas à tout le monde. $\mathrm{Au}$ Mexique, pays de forte émigration, seuls $4,35 \%$ des ménages en reçoivent ${ }^{24}$. D'après la même source, certains Etats présentent des pourcentages de $13 \%$ de ménages bénéficiaires, comme Zacatecas, ou de $11 \%$, comme Michoacan, mais dans tous les autres Etats ce taux se situe en dessous de $10 \%$. On note une différence entre le milieu rural (10\% environ) et le milieu urbain (4\%).

Au niveau local, les études empiriques concernent surtout l'Etat de Zacatecas, au Mexique, région de vieille émigration aux Etats-Unis ${ }^{25}$; quant aux autres pays, comme la forte vague de migration ne date que des années 1990, nous avons à notre disposition un matériel très restreint. Les récentes études systématisées du Groupe Chorlavi fournissent cependant un matériel sérieux pour la Colombie, l'Equateur, le Pérou et la Bolivie, mais toujours en nombre limitéé Ces études touchent essentiellement le milieu rural, et c'est donc sur lui que nous nous concentrerons.

Une première remarque s'impose: l'utilisation des fonds envoyés par les migrants montre de grandes similitudes entre les régions et pays. La majeure partie de ces fonds (un bon tiers) sert en effet à lutter contre la pauvreté et est donc dépensée en nourriture, habillement et logement. Viennent ensuite les dépenses de santé (entre $20 \%$ et $25 \%$ ) puis celles pour l'éducation des enfants (en proportion un peu moindre). Les trois postes additionnés représentent $80 \%$ du total reçu, ce qui mène certains économistes et analystes à conclure qu'il s'agit là essentiellement de dépenses de consommation. Or, certaines dépenses ménagères peuvent être comprises comme des investissements car, comme l'a montré Jim Airola pour le cas du Mexique ${ }^{27}$, il faut séparer les biens ménagers non durables des durables, tels les biens électroménagers qui, hormis la télévision et la radio, peuvent être considérés comme des biens d'investissement. A partir des données d'une enquête de ménage menée de 1984 à $2000^{28}$, l'auteur

23 Roxana Dulón, op. cit.

24 Claudia Meza Merlos y Humberto Márquez Covarrubias, «Cambios en el patrón migratorio y pobreza en Zacatecas », in Raúl Delgado Wise y Beatrice Knerr (coord.), op. cit., p. 251 tableau 3, d'après le $12^{\mathrm{e}}$ recensement de la population (2000) du Mexique.

25 Rodolfo Garcia Zamora, Migración, remesas y desarrollo, los retos de las organizaciones migrantes mexicanas en Estados Unidos, México, Universidad Autónoma de Zacatecas, 2005. Volker Hamann, The Impact of International Labor Migration on Regional Development: The Example of Zacatecas, Mexico, Kassel; Kassel University Press, 2006. Raúl Delgado Wise y Beatrice Knerr (coord.), op. cit.

26 Le Groupe Chorlavi est une organisation non gouvernementale de recherche et d'action sur la petite paysannerie, qui a son siège à Santiago du Chili. Il a mené des études approfondies sur les changements dans les agricultures andines et sur les migrations. Il prône une agriculture écologique et de proximité. Voir <http://www.grupochorlavi.org $>$.

27 Jim Airola, op. cit.

28 Instituto Nacional de Estadisticas, Geografia e Informatica (INEGI), Encuesta Nacional de Ingresos y Gastos de Hogares, Mexico, 2000. 
constate que les modèles de consommation des ménages de migrants diffèrent de ceux des non-migrants: ils investissent davantage dans des biens domestiques durables $(+56 \%)$ ainsi qu'en dépenses de santé $(+44 \%)$ et d'entretien ménager $(+17 \%)$. Par contre, l'enquête ne montre pas de grandes différences entre catégories quant aux dépenses d'éducation et une baisse relative dans les dépenses d'alimentation ( $-8 \%$ ) pour les migrants ${ }^{29}$.

Les dépenses de santé sont en partie des dépenses d'investissement humain lorsqu'il s'agit de prévention, de vaccination des enfants, etc. De même, les dépenses d'éducation, qui vont former du capital humain, constituent un investissement. Les familles rurales et urbaines recherchent souvent de bons collèges pour leurs enfants, quitte à les envoyer dans une autre localité ou à déménager en bloc ${ }^{30}$. De nombreuses enquêtes rurales dans les Andes montrent que l'accumulation d'argent par les paysans sert avant tout à favoriser l'avenir de leurs enfants, les parents considérant la plupart du temps qu'ils ne pourront pas progresser eux-mêmes beaucoup plus ${ }^{31}$.

Quant aux 20\% restants, ils se répartissent parfois différemment selon les régions, mais servent essentiellement à l'amélioration physique de la maison ou à l'achat d'une nouvelle demeure et de terres, au paiement des dettes et à l'épargne bancaire (entre $3 \%$ et $5 \%$ ).

\section{Les investissements financiers}

La faible part (5\%) consacrée à l'épargne bancaire renforce généralement l'opinion que l'épargne et donc l'investissement sont peu favorisés par les migrants et les institutions financières. Il est vrai que les migrants ont une «préférence pour la liquidité » et répugnent à passer par des systèmes bancaires, souvent parce qu'ils séjournent dans le pays d'accueil de façon illégale. Mais les études montrent que les dépôts bancaires individuels réalisés ponctuellement sont souvent substantiels, de l'ordre de 1000 dollars et plus ${ }^{32}$, ce qui représente un capital important pour des milieux pauvres. Les migrants procèdent fréquemment à des envois extraordinaires de fonds, de l'ordre de plusieurs milliers de dollars. En outre, l'achat d'un terrain ou la construction d'une maison sont aussi des investissements.

A quels critères répond cette distribution des transferts? Comme on l'a vu plus haut, c'est la consolidation physique des familles qui forme la base des comportements, avec des dépenses pour le ménage, la santé et les enfants, ainsi que pour un confort accru.

29 En fait leurs dépenses de consommation en valeur absolue augmentent aussi, mais dans une moindre mesure.

30 Geneviève Cortes, Partir pour rester: survie et mutation des sociétés paysannes (Bolivie), coll. «A travers champs», Paris, Institut de recherche sur le développement (IRD), 2000. Gloria Camacho y Kattya Hernández, Territorios entrelazados: Impactos de la emigracion internacional para el desarrollo rural de Suscal, Quito, Instituto de estudios ecuatorianos, Grupo Chorlavi, 2008

31 Claude Auroi, «Les agricultures andines, une lente évolution», in Claude Auroi et Jean-Luc Maurer, Tradition et modernisation des économies rurales: Asie-Afrique-Amérique latine. Mélanges en l'honneur de Gilbert Etienne, Paris, Presses Universitaires de France, 1998, pp. 285-309. Claude Auroi, «Situación alimenticia en América Latina y futuro de la producción agropecuaria para pequeños campesinos », Colloque CEISAL, avril 2007, Bruxelles (à paraître en 2008).

32 Gloria Camacho y Kattya Hernández, op. cit. 
Les dépenses de reproduction concernent l'acquisition de capital physique biens-fonds, terrains, animaux, construction de puits et de canaux d'irrigation, achat de véhicules, etc. ${ }^{33}$ - mais elles touchent aussi une meilleure éducation pour les enfants.

Enfin, une part des dépenses a trait au maintien ou au renforcement du capital social dans les communautés, par des apports ponctuels à des œuvres ou des constructions, par exemple des églises, des écoles, des fêtes annuelles et des cadeaux $^{34}$.

Cette stratégie paraît cohérente et même avisée lorsque l'on connaît les structures sociales et de pouvoir relativement rigides des communautés rurales en Amérique latine. Elle répond aux nécessités du moment (lutter contre sa propre pauvreté et affirmer son identité) et à celles du futur (assurer sa vieillesse et l'avenir des nouvelles générations). Même s'il n'est pas forcément recherché, le prestige social suscité par le processus de redistribution collective d'une partie des ressources gagnées a son utilité. Garder des liens communautaires est essentiel car la migration peut être volatile, et donc le retour nécessaire un jour ou l'autre.

Cette stratégie est-elle durable? On ne peut répondre à cette question sans considérer si la migration en elle-même est durable, car il est évident que pour consolider sur place une famille pauvre il faut l'apport des fonds des migrants sur de nombreuses années. Dans la mesure où ceux-ci sont jeunes (ils ont entre 20 et 40 ans), le phénomène des transferts de fonds devrait se poursuivre dans sa forme actuelle pendant une bonne génération; il s'agit donc d'un investissement économiquement durable.

\section{Efforts pour drainer les fonds des migrants vers des investissements collectifs}

Miguel Moctezuma Longoria distingue cinq types de migrant investisseur: le migrant collectif, le migrant entrepreneur, le migrant épargnant, le migrant qui survit et le migrant à la retraite et de retour au pays ${ }^{35}$.

C'est le migrant collectif qui retient surtout notre attention ici, bien qu'il se confonde parfois avec le migrant entrepreneur. Ce dernier a généralement réussi à créer un commerce dans le pays d'accueil, par exemple un restaurant, une boutique avec les produits du pays ou un magasin de vêtements. Le troisième type de migrant épargne généralement pour s'acheter une maison ou un terrain. Le migrant qui survit n'a pas les moyens d'investir et le retraité va parfois ouvrir un petit commerce ou tenir un kiosque.

Le migrant collectif participe à un effort coordonné pour apporter une aide à sa communauté de départ. Le Mexique est un des pays avec le Salvador dont le gouvernement a tenté de drainer et canaliser certains de ces flux. Ce furent les

33 Rodolfo Garcia Zamora a étudié l'investissement technique dans les fermes de l'Etat de Zacatecas, en différenciant les exploitations tenues par des anciens migrants et celles tenues par des non-migrants. Les premières sont plus souvent capitalisées (camionnettes, tracteurs, charrues tractées, cultivateurs, etc.). Rodolfo Garcia Zamora, op. cit., p. 62, tableau 16.

34 Geneviève Cortes, op. cit.

35 Miguel Moctezuma Longoria, «La cultura migrante y el simbolismo de las remesas. Reflexiones a partir de la experiencia de Zacatecas», in Raúl Delgado Wise y Beatrice Knerr (coord.), op. cit., p. 119. 
programmes Dos por uno et Tres por uno qui, dès les années 1993 et 1999 respectivement, suscitèrent des partenariats entre les associations ou clubs de migrants aux Etats-Unis et leurs Etats et communautés de départ (Dos por uno) et ensuite avec l'Etat fédéral comme troisième partenaire ${ }^{36}$. Les associations apportent une part d'une somme qui est triplée par les pouvoirs publics pour la construction ou l'entretien d'infrastructures allant des réseaux d'eau aux bâtiments scolaires ou aux églises. Les sommes ainsi récoltées sont assez importantes et constituent une aide sans laquelle des collectivités auraient quelque difficulté à assurer leur subsistance. Certains auteurs ont argumenté que l'Etat se voyait ainsi soulagé en partie dans ses investissements, ce qui entrait dans la logique de la nouvelle gouvernance prônée par le néolibéralisme ${ }^{37}$. Quoi qu'il en soit, ce modèle a été repris dans d'autres pays d'Amérique centrale, mais il peine à s'implanter en Amérique du Sud.

Une autre critique à l'encontre de ce modèle est qu'il n'a guère contribué à créer des entreprises productives, à stimuler l'innovation et l'esprit entrepreneurial ${ }^{38}$. Il est vrai qu'il est plus facile de créer une belle place publique que de développer des entreprises, bien que les potentialités soient présentes dans plusieurs domaines $^{39}$. Le cas du village de Atacheo de Regalado, au Michoacan, est particulièrement révélateur du manque de capacité à gérer. Dans ce village, le curé avait monté trois projets avec une mise de fonds de la part des migrants: la fabrication d'instruments pour les groupes musicaux, l'élevage de dindons et l'élevage caprin. Les trois projets se sont écroulés, et les trois à cause d'une mauvaise gestion et de pertes financières (celui des dindons aussi en partie pour des raisons épidémiques $)^{40}$.

Le capital humain reste à former pour qu'il puisse utiliser le capital financier à bon escient et s'intégrer dans des filières. A petite échelle, ces efforts existent déjà, notamment chez les producteurs qui se sont spécialisés dans l'envoi de denrées et articles aux communautés émigrées. Dans toutes les villes des Etats-Unis et d'Europe où existent de grandes communautés, on peut trouver des magasins de produits mexicains, péruviens, boliviens, etc. Sur cette base, il est certainement possible à l'avenir d'étendre les activités productives à des produits plus sophistiqués incorporant plus de valeur ajoutée. Mais pour ce faire il faut aussi

36 Rafael Fernández de Castro, Rodolfo Garcia Zamora y Ana Vila Freyer (coord.), El programa 3x1 para migrantes. ¿Primera política transnacional en Mexico?, México, Universidad Autónoma de Zacatecas, 2006. Au début le programme s'appelait Uno por uno, l'association des migrants et l'Etat de Zacatecas mettant chacun une part. On a même vu une tentative de Cuatro por uno, avec la participation de Western Union (Western Union, Western Union Expands Initiative to Promote Community Economic Development in Mexico; Mexican State of Michoacan the Second State to benefit from $4 x 1$ Program, Press Release, 23 June 2006, <http://ir.westernunion.com/investor/releasedetail.cfm?Release $\mathrm{ID}=219081>$ ).

37 Alejandro I. Canales, «Envois de fonds, développement et pauvreté, une perspective critique de l'Amérique latine», in Isabel Yépez et Gioconda Herrera (dir.), op. cit., pp. 195-217.

38 Le programme Tres por uno, entre 2002 et 2005, n'a financé que $4 \%$ de projets productifs proprement dits, le reste étant destiné aux infrastructures (Rafael Fernández de Castro, Rodolfo Garcia Zamora y Ana Vila Freyer [coord.], op. cit., pp. 17-18).

39 Alejandrino S. Loaiza, L. Herrea y César Sotomayor, op. cit.

40 Fernando Neira Orjuela, Sistematización de las experiencias en el uso productivo de remesas para el fomento del desarrollo: un estudio de caso en proyectos productivos del municipio de Atacheo de Regalado, en el Estado de Michoacán de Ocampo, México, México, UNAM y Grupo Chorlavi (Fondo Mink'a), 2008. 
renforcer les réseaux bancaires et financiers travaillant avec les migrants et les bénéficiaires des envois de fonds.

\section{Les effets économiques et sociaux des envois de fonds}

L'envoi massif de fonds dans des milieux pauvres a des effets contrastés sur le milieu social, avec des aspects bénéfiques pour les bénéficiaires mais aussi parfois «perturbateurs » pour le milieu social. C'est dans les petites communautés à forte émigration (parfois $50 \%$ de la main-d'œuvre) que l'impact de la migration est le plus visible.

\section{La diminution de la pauvreté}

Les études de la Banque mondiale ${ }^{41}$ et d'autres institutions montrent que les transferts de fonds vont vers les quintiles les plus pauvres de la population: «Par exemple au Mexique, les bénéficiaires des envois sont en majorité pauvres: $61 \%$ des ménages déclarant recevoir des envois font partie du premier quintile des revenus (sans compter les envois), alors que seuls $4 \%$ d'entre eux sont dans le quintile le plus élevé. ${ }^{42}$ En général, les pays d'Amérique du Sud présentent la même distribution «propauvres », à l'exception du Pérou où les ménages du premier quintile reçoivent $6 \%$ des remises, le quintile le plus élevé touchant quant à lui $40 \%$ du total; on remarque la même distribution inégalitaire au Nicaragua. Dans ces deux pays, ce sont des familles plus aisées qui émigrent. Dans quatre pays, la distribution entre les groupes de revenu est plus ou moins égale entre les groupes riches et pauvres (Bolivie, Honduras, République dominicaine et Haïti) ${ }^{43}$.

\section{La réduction des inégalités}

Si l'effet «antipauvreté» est avéré pour les destinataires des envois, celui de la réduction des inégalités est plus ambigu. Il doit être replacé dans un contexte global où finalement un nombre important de familles ne sont pas bénéficiaires.

Lorsque l'on étudie l'effet de la répartition des transferts de fonds sur la distribution totale des revenus (transferts compris), on s'aperçoit que la part des plus pauvres diminue fortement dans tous les pays et qu'à l'exception du Mexique, du Paraguay et du Salvador, plus de la moitié des bénéficiaires d'envois se retrouvent désormais dans les deux quintiles supérieurs; concrètement, cela veut dire que les bénéficiaires ont fait un saut vers le haut dans l'échelle des revenus du pays. Cela est particulièrement le cas des pays dont les émigrants appartiennent plutôt aux classes supérieures (Pérou). Mais même lorsqu'il y a égalité de revenu au départ entre migrants et non-migrants, comme à Sisid, en Equateur, les familles de migrants gagnent soudainement $50 \%$ de plus que celles restées sur place ${ }^{44}$. Il s'en-

${ }^{41}$ World Bank, The Development Impact of Workers' Remittances in Latin America, op . cit.

42 Pablo Fajnzylber and J. Humberto López, op. cit., p. 6 (NDA: notre traduction).

43 Ibid., p. 6.

44 Centro de Desarrollo e Investigación Rural (CEDIR), Migración internacional en la comuna Sisid: cambios y adaptaciones en el territorio rural y economia local, Quito, CEDIR y Grupo Chorlavi (Fondo Mink'a), 2008, graphique 7. 
suit, d'après la Banque mondiale, que si les envois de fonds de migrants sont un facteur indéniable de réduction de la pauvreté, ils ne jouent pas le même rôle dans la réduction des inégalités, qu'ils auraient plutôt tendance à augmenter ou à maintenir dans le statu quo ${ }^{45}$.

Ces constatations globales sont fondamentales au niveau macrosocial, mais doivent être nuancées selon les pays, les régions et au niveau local. Les modèles de migration diffèrent fortement d'une région à l'autre, selon le groupe social, le genre, le pays et l'occupation de destination, les montants envoyés à la famille et l'usage qui en est fait. Les études dont on dispose montrent que les inégalités augmentent dans les zones de forte migration.

Par exemple à Sisid, en Equateur, les habitants peuvent être classés nouvellement en

$1^{\circ}$ bénéficiaires de sommes très élevées (en général dans le cas de l'émigration du mari);

$2^{\circ}$ bénéficiaires de sommes moyennes (dans le cas de l'émigration d'un ou plusieurs enfants) et «professionnels » restés sur place;

$3^{\circ}$ familles restées sur place;

$4^{\circ}$ familles ayant perdu un membre émigré qui la soutenait et vivant dans l'extrême pauvreté ${ }^{46}$.

Mais il existe aussi des cas où la conséquence de l'émigration peut être un statu quo local relatif dû au fait que les émigrants préfèrent investir hors du village, dans la ville proche, comme en Bolivie ${ }^{47}$. Ce choix révèle une volonté d'ascension sociale planifiée, souvent liée à la recherche de meilleurs collèges pour l'éducation des enfants.

\section{Le marché du travail}

Le départ des hommes de la classe d'âge de 20 à 40 ans provoque un manque de bras qui ne peut être toujours compensé par le travail féminin ou l'arrivée d'immigrés nationaux ou étrangers. S'ensuivent l'abandon de certaines tâches agricoles et la concentration des activités de ce secteur, notamment dans l'élevage. En outre, l'offre de travail s'accroît dans d'autres domaines d'activité, principalement la construction, ce qui réduit encore l'offre dans l'agriculture. Des terres restent ainsi à l'abandon ou sont louées à d'autres propriétaires ; certains terrains sont vendus.

Les sommes parfois importantes envoyées par les migrants peuvent aussi être un facteur de diminution du temps de travail ou de moindre participation au marché du travail. Les enquêtes de Pablo Fajnzylber et J. Humberto López montrent que dans chacun des dix pays de l'échantillon retenu, le nombre d'heures de travail des membres des familles de migrants est en baisse, aussi bien dans les villes que dans les milieux ruraux et pour les deux $\operatorname{sexes}^{48}$. La participation au marché

45 World Bank, The Development Impact of Workers' Remittances in Latin America, op. cit.

46 Centro de Desarrollo e Investigación Rural (CEDIR), op. cit.

47 Geneviève Cortes, op. cit. Roxana Dulón, op. cit.

48 Pablo Fajnzylber and J. Humberto López, op. cit., p. 31. 
du travail est aussi plus réduite, à l'exception du Mexique, du Honduras, du Guatemala et de la République dominicaine. Le taux d'incorporation au marché est plus élevé pour les personnes ayant pu suivre l'école primaire pendant au moins quatre ans. Jim Airola arrive à la conclusion que le nombre d'heures de travail est en baisse au Mexique à la suite de la réception des fonds ${ }^{49}$. Ezra Rosser estime que cet effet revenu pervers peut cependant être positif si les salaires s'élèvent dans la région et constituent un stimulant pour l'emploi - pour autant qu'ils s'élèvent assez pour compenser les avantages du temps libre généré par les fonds reçus des migrants ${ }^{50}$. Les conséquences sociales de la nonintégration au marché du travail peuvent aussi consister dans un accroissement de la violence de la part de jeunes désœuvrés, qui pourraient former des bandes sur le modèle des maras d'Amérique centrale ${ }^{51}$.

\section{Emigration et développement régional}

Alors que les transferts de fonds devraient normalement permettre de stabiliser les populations pauvres dans leur milieu, les «nouveaux riches » cherchent souvent au contraire à quitter leur pueblo (village) pour s'établir dans des centres régionaux plus attractifs. Comme le montre Beatrice Knerr, les régions de départ des migrants sont souvent parmi les plus dénuées d'infrastructures et de possibilités d'emploi. Dès lors, les personnes qui restent sur place, si elles veulent travailler, vont également émigrer vers les chefs-lieux, intensifiant la désertion des villages plus ou moins abandonnés du fait de la migration. En ce qui concerne l'emploi, l'auteure cite des exemples de l' «effet Ravenstein», soit le remplacement des migrants par des immigrants d'autres régions ou pays, comme de l'Ukraine vers la Pologne, de l'Egypte vers la Jordanie ou du Guatemala vers le Chiapas, au Mexique ${ }^{52}$. Il en va de même en Equateur, où les ouvriers agricoles péruviens remplacent les émigrés locaux. Quant aux émigrés «du retour», ils chercheront aussi à s'établir dans des endroits plus attrayants sur les plans économique et socioculture ${ }^{53}$. Les raisons de ces transferts de domicile sont souvent liées à la recherche de meilleures possibilités de scolarisation pour les enfants des migrants; or, les collèges de niveau supérieur, souvent privés, se trouvent dans les centres urbains.

A propos de l'éducation, signalons que les répercussions des fonds reçus des migrants sur la scolarisation restent limitées. On a pu noter une moindre désertion scolaire chez les plus pauvres, mais pas forcément de meilleurs résultats pour les enfants des migrants en général ${ }^{54}$.

La compensation de la perte d'emplois par la création d'activités grâce aux fonds envoyés par les migrants reste limitée à une certaine demande locale en

49 Jim Airola, op. cit.

50 Ezra Rosser, op. cit.

51 Ibid., p. 29.

52 Beatrice Knerr, «Dinàmicas económicas regionales frente a la migración laboral internacional. Teorías y experiencias globales », in Raúl Delgado Wise y Beatrice Knerr (coord.), op. cit., pp. $137-$ 170, p. 146. Ernest George Ravenstein émit sa théorie sur la substitution des migrations entre 1885 et 1890 déjà.

53 Ibid., p. 147

54 Pablo Fajnzylber and J. Humberto López, op. cit. 
produits de consommation et, dans le cas des investissements collectifs, à la construction d'infrastructures.

\section{Les structures sociales et de pouvoir}

Une autre question qui se pose sur les effets de la migration au niveau local est la suivante: les transferts de fonds ont-ils des répercussions économiques à même de mettre en cause l'organisation sociale des communautés bénéficiaires? Y a-t-il rupture des hiérarchies et des solidarités traditionnelles, ou les sociétés locales sont-elles en mesure d'intégrer les changements économiques sans bouleversements sociaux?

Les trop rares études à disposition laissent entrevoir que la structure sociale des villages à forte migration est peu à peu érodée par l'argent, qui crée de nouveaux groupes de pouvoir ou fragmente les communautés; il y a donc bien une recomposition sociale, qui dépend beaucoup du pays considéré et de l'ampleur de la migration.

Dans les Etats où les populations sont très métissées depuis longtemps, comme le Salvador, la République dominicaine ou le Mexique, les fonds reçus des migrants ont un effet essentiellement social, dans la mesure où ils permettent à des classes sociales jusque-là méprisées d'obtenir un empowerment certain. Le cas des «artistes de cabaret» dominicaines en Suisse, qui ont envoyé énormément d'argent à leurs parents, est symptomatique de cette reconsidération sociale pour des familles et des personnes situées au bas de l'échelle sociale mais qui ont financièrement réussi ${ }^{55}$. Dans ce cas, l'aspect moral ne joue pas un grand rôle, probablement parce que cette société était déjà assez permissive au départ.

Dans les pays andins (Equateur, Pérou, Bolivie), il apparaît que la structure sociale de départ est plus rigide et que les rapports de hiérarchie tendent à résister au changement. C'est surtout le cas des relations de genre: même en travaillant à des milliers de kilomètres, les hommes cherchent à garder le pouvoir de décision dans les affaires communales et dans la vie familiale, sans le céder aux femmes restées sur place ${ }^{56}$. Dans la communauté de Sisid, les indigènes conduisent les ex-hacendados (propriétaires d'haciendas) à renoncer au pouvoir communal, ce qui a incité certains métis à quitter totalement la communauté. Ces mutations et cette ascension sociale dépendent des contextes spécifiques, de la force relative des groupes en présence. Les enquêtes sont trop partielles pour dégager des tendances générales dans le monde andin.

Avec le phénomène des transferts de fonds, on assiste donc indéniablement en Amérique latine à un profond bouleversement des structures sociales des communautés rurales, qui se manifeste par des tensions qui vont remodeler le pouvoir politique local et probablement au-delà. Ce phénomène est cependant concomitant d'autres chocs sociaux et culturels que subissent les campagnes, liés à l'irruption du tourisme, aux nouvelles techniques de culture (aspersion) et

55 Jennifer Petree y Tahira Vargas, Dominicanos en Suiza. Pautas, practicas e impactos de la migración transnacional y el envió de remesas que vinculan Republica Dominicana y Suiza, EPFL, Cahiers du LaSUR, nº 8, Lausanne, Ecole polytechnique fédérale de Lausanne, 2005.

56 Nous revenons plus loin sur les relations de genre. 
d'élevage (embouche) et à l'amélioration des infrastructures qui rapprochent les villages des centres urbains.

La création de «nouveaux riches» ne signifie cependant pas la réduction des inégalités car parallèlement apparaissent de «nouveaux pauvres» qui sont, dans les villages, les exclus de la migration ou ceux qui ont perdu leur soutien extérieur, généralement à la suite d'un décès. Les structures sociales sont donc en recomposition là où la migration est forte, et elles le sont moins dans les régions à forte dominance de grands propriétaires terriens bien établis et contrôlant le pouvoir depuis des décennies, comme au Chiapas ou à Oaxaca, au Mexique. Dans les villes, les migrants qui ont réussi se dissolvent dans une masse urbaine trop dense pour que des effets importants se fassent sentir.

\section{Envois de fonds et ethnicité}

La relation entre les facteurs ethniques et la migration a été peu étudiée. Lors d'une enquête dans quelques communautés indigènes à forte émigration de l'Etat d'Oaxaca, on a remarqué que les fonds reçus des migrants sont utilisés pour le développement local de manière collective, en passant par les mécanismes du contrôle communal traditionnel. Il apparaît comme un devoir moral et social aux yeux du migrant de contribuer à des investissements publics, mais les décisions sont prises par l'assemblée communale, et non par les migrants eux-mêmes. Ceux-ci doivent aussi théoriquement participer aux charges communales (travaux divers) et assumer des postes d'édile si la communauté le demande. Dans ces cas cependant, le migrant qui se trouve trop éloigné, en Californie par exemple, peut payer un remplaçant, mais il sera alors responsable des actions de ce dernier ${ }^{57}$. Les sanctions des infractions sont sévères: elles vont de la confiscation des terres au retrait de l'identité communautaire et au bannissement.

Dans les Andes, on note un déplacement des élites métisses par les migrants indigènes qui de ce fait ont pris de l'assurance et se sont enrichis. En Equateur, les indigènes acquièrent une plus grande mobilité sociale ${ }^{58}$. Mais le peu d'études dont on dispose ne permettent pas de conclure si les traditions communautaristes vont être renforcées ou affaiblies. Dans tous les cas, les transferts de fonds restent essentiellement familiaux, quelle que soit l'appartenance ethnique. Le contrôle collectif se fait surtout sur les fonds additionnels attribués aux communautés. Mais on peut faire l'hypothèse qu'au vu des changements économiques et de l'enrichissement relatif de certaines familles, des tensions sociales vont surgir dans les communautés indigènes et que le système coutumier de gouvernance en sera affecté.

57 Beatriz Straffon, Paola Lopez y Anally Castellanos, «El Programa 3x1 para Migrantes en Oaxaca. Migracion indigena, practicas comunitarias y costumbres de participacion», in Rafael Fernández de Castro, Rodolfo Garcia Zamora y Ana Vila Freyer (coord.), op. cit., pp. 197-223.

58 Centro de Desarrollo e Investigación Rural (CEDIR), op. cit. Gloria Camacho y Kattya Hernández, op. cit. 


\section{Les dépenses somptuaires et de prestige}

Les migrants manifestent parfois une propension aux dépenses somptuaires et de prestige, inutiles dans une perspective de développement, mais souvent utiles au maintien du capital social du migrant.

Sous la pression communautaire ou de sa propre volonté, le migrant - on l'a dit plus haut - cherche à maintenir sa position sociale par des apports ponctuels à des œuvres ou des constructions, par exemple des églises, des écoles, des fêtes annuelles, et par l'apport de cadeaux lorsqu'il revient une fois par $a^{59}$. La construction d'une maison familiale est particulièrement prisée, car celle-ci, outre le fait qu'elle améliore les conditions de vie, répond aussi à des considérations de prestige, par la création d'un capital symbolique ${ }^{60}$. La nouvelle demeure doit dès lors répondre à des critères de modernité en rupture avec le style classique des maisons des bourgades et inspirés par les modèles des pays d'accueil, de magazines immobiliers ou de séries télévisées. De ce fait, les constructions des «nouveaux riches» présentent souvent un caractère extravagant, kitsch et pas forcément fonctionnel. Le tissu architectural et urbanistique traditionnel en est grandement affecté. On assiste dans les Andes à une lente dégradation du patrimoine architectural colonial, qui n'est d'ailleurs pas le fait exclusif de la migration et du mauvais goût, mais aussi de l'absence de planification urbaine.

L'effet de consommation somptuaire ne doit cependant pas être exagéré, car les fonds reçus ne signifient pas l'abondance, et la majorité des dépenses sont de consommation courante. Comme le note Manuel Orozco: «The cost of living in Latin American countries is at least two or three times the average amount received in remittances. ${ }^{61}$

\section{Les différences de genre}

Une fois leurs maris partis, les femmes sont amenées à accomplir des tâches qui ne leur étaient pas dévolues auparavant, comme de lourds travaux agricoles (par exemple la préparation des terres), du commerce, des activités dans la politique communale, etc. Il en résulte souvent une surcharge du travail féminin, qui ne peut pas toujours être compensée par l'aide familiale ou le travail salarié.

Les femmes se trouvent souvent en porte-à-faux entre des exigences nouvelles nécessitant de leur part un pouvoir de décision et l'opinion de leurs maris, qui restent en contact téléphoniquement. Une enquête auprès de 299 émigrés de la ville de Jerez, dans l'Etat de Zacatecas au Mexique, a montré que la fréquence des contacts téléphoniques est élevée: $50 \%$ des migrants téléphonent au moins une fois par semaine et $18 \%$ une fois toutes les deux semaines; moins de $1 \%$ seulement ne téléphonent jamais ${ }^{62}$. A Sisid, en Equateur, pendant les assemblées communales, les femmes sont en relation téléphonique avec leurs maris émigrés aux Etats-Unis afin de recevoir des instructions lors des votes... ${ }^{63}$ Dans le cas

\footnotetext{
Geneviève Cortes, op. cit.

60 Jennifer Petree y Tahira Vargas, op. cit.

61 Manuel Orozco, op. cit.

62 Voir Rodolfo Garcia Zamora, op. cit., p. 159, tableau 8.

63 Roxana Dulón, op. cit.
} 
des pays proches des Etats-Unis, tel le Mexique, il faut aussi considérer que la fréquence des retours au pays est relativement élevée, une fois tous les deux ans pour certains villages de Zacatecas ${ }^{64}$. Cependant, la même enquête montre également que la moitié des émigrés ne reviennent pas du tout et se contentent d'envoyer les fonds.

\section{Conclusions}

La migration entraîne certes un certain nombre d'effets «pervers » ou perturbateurs au niveau local et régional, mais ceux-ci doivent être contrebalancés par les aspects positifs suivants: réduction globale de la pauvreté dans les communautés à forte émigration, amélioration du confort ménager et meilleures conditions d'hygiène et de santé, capitalisation relative des entreprises agricoles (machines, puits, moyens de transport) et agrandissement des propriétés.

Les fonds de la migration constituent aussi une prévoyance et une sécurité sociale que l'Etat ne peut fournir en Amérique latine. Les retraites des parents sont assurées par la migration des enfants et des parents eux-mêmes pour leurs vieux jours. La «famille transnationale» agit donc à tous les niveaux pour assurer la survie présente et intergénérationnelle. Mais de ce fait même la pression sur l'Etat est diminuée, empêchant l'avènement de régimes sociaux basés sur la taxation et l'épargne sociale. Le terme de «famille transnationale» a donc aussi ses ambiguïtés. Il désigne un modèle de mondialisation établie et relativement solide, mais dans et entre des Etats qui eux ne sont pas transnationaux puisque dans la plupart des cas ils n'ont pas coordonné leurs politiques sociales. Pour les migrants clandestins, la situation est aggravée par le fait qu'ils ne sont pas inscrits dans les systèmes d'aide sociale ou de pension.

Il serait erroné d'affirmer que les mouvements migratoires de travailleurs du Sud vers le Nord sont du pain bénit ou une manne tombée du ciel pour les parents restés au pays. La séparation des familles crée parfois de graves problèmes dans les communautés de départ; les femmes notamment, dont les maris sont absents plusieurs mois, voire plusieurs années, ont souvent de la peine à devoir faire face à des problèmes de gestion familiale, de travail des champs et également de participation communale. Cette surcharge de travail peut mener à des abandons, des séparations ou des divorces, ou au regroupement des conjoints à l'étranger qui laisse entier le problème de l'éducation et de l'affection témoignée aux enfants laissés aux bons soins des grands-parents, des tantes et des oncles.

Néanmoins, le fait migratoire en Amérique latine est considéré comme positif par les agences internationales et nationales car il permet de faire bouger des sociétés jusque-là maintenues dans un état de soumission et d'immobilisme par manque de moyens. La tâche consiste donc non pas à refuser le «fait migratoire et les fonds de la migration», mais à aménager ces deux mouvements afin qu'ils profitent le mieux possible à ceux qui en sont les générateurs et les bénéficiaires, ainsi qu’à leurs communautés. Il faut encourager les Etats et les orga-

64 Volker Hamman, «Diferencias intraregionales en la zona de alta migración zacatecana», in Raúl Delgado Wise y Beatrice Knerr (coord.), op. cit., pp. 193-206, p. 199, tableau 4. 
nismes internationaux à entrer dans le jeu comme partenaires de développement, en apportant leur part financière et leur expertise (uno, dos, tres, cuatro por uno). Les programmes de microcrédit pourraient notamment être reliés à la mobilisation des fonds de la migration.

Le fait migratoire montre que «les pauvres aident les pauvres» au niveau personnel et familial bien mieux que lorsque les riches s'en mêlent. Rappelons que les montants des fonds privés envoyés par les migrants sont supérieurs aux investissements extérieurs directs. Cela ne veut pas dire que ces fonds puissent remplacer l'action de l'Etat et des aides extérieures, mais cela montre que dans l'équation du développement, de nouveaux partenaires, les migrants, doivent être pris en compte et totalement intégrés dans le processus de réflexion et de mise en œuvre des projets.

\section{Bibliographie}

Airola, Jim, «Labor Supply in Response to Remittance Income: The Case of Mexico», The Journal of Developing Areas, vol. 41, n 2, Spring 2008.

Airola, Jim, «The Use of Remittance Income in Mexico (Report)», International Migration Review, vol. $41, n^{\circ} 4,2007$.

Amuedo-Dorantes, Catalina and Susan Pozo, «On the Use of Differing Money Transmission Methods by Mexican Immigrants », International Migration Review, vol. 39, n 3, 2005.

Auroi, Claude, «Cambios en la pequeña agricultura andina: hacia la intensificación. Una encuesta a tres comunidades en el Departamento de Cusco, Perú », Congreso CEISAL Bratislava, 5-7 de julio de 2004 (inédit).

Auroi, Claude, «Situación alimenticia en América Latina y futuro de la producción agropecuaria para pequeños campesinos », Colloque CEISAL, avril 2007, Bruxelles (à paraître en 2008).

Auroi, Claude et Jean-Luc Maurer, Tradition et modernisation des économies rurales: Asie-AfriqueAmérique latine. Mélanges en l'honneur de Gilbert Etienne, Paris, Presses Universitaires de France, 1998, 394 p.

Auroi, Claude et Isabel Yépez del Castillo (dir.), Economie solidaire et commerce équitable: acteurs et actrices d'Europe et d'Amérique latine, Genève, Institut universitaire d'études du développement (IUED); Louvain, Presses Universitaires de Louvain, 2006.

Auroi, Claude, «Les envois de fonds pour le développement local. Réflexions à partir de cas latinoaméricains », in Isabel Yépez et Gioconda Herrera (dir.), Nouvelles migrations latino-américaines en Europe, Barcelona, Publicacions i Edicions de la Universitat de Barcelona, pp. 169-194.

Banco Central de Reserva de El Salvador, Metodología de calculo e importancia de las remesas familiares en El Salvador, 2004, <http://www.cemla-remesas.org/PDF/seminariomx/0510MX-ES-PPTElSalvador.pdf $>$.

Bendixen and Associates, Remittances and the Dominican Republic: Survey of Recipients in the Dominican Republic, Survey of Senders in the United States, November 2004, <http://www.iadb.org/NEWS/ docs/SurveysRemittanceFlows_e.ppt>.

Centro de Desarrollo e Investigación Rural (CEDIR), Migración internacional en la comuna Sisid: cambios y adaptaciones en el territorio rural y economia local, Quito, CEDIR y Grupo Chorlavi (Fondo Mink'a), 2008.

Camacho, Gloria y Kattya Hernández, Territorios entrelazados: Impactos de la emigracion internacional para el desarrollo rural de Suscal, Quito, Instituto de estudios ecuatorianos, Grupo Chorlavi, 2008.

Canales, Alejandro I., «Envois de fonds, développement et pauvreté, une perspective critique de l'Amérique latine», in Isabel Yépez et Gioconda Herrera (dir.), Nouvelles migrations latino-américaines en Europe, Barcelona, Publicacions i Edicions de la Universitat de Barcelona, pp. 195-217.

Cortes, Geneviève, Partir pour rester: survie et mutation des sociétés paysannes (Bolivie), coll. «A travers champs », Paris, Institut de recherche sur le développement (IRD), 2000.

Delgado Wise, Raúl y Beatrice Knerr (coord.), Contribuciones al análisis de la migración internacional y el desarrollo regional en México, Universidad Autónoma de Zacatecas, 2005. 
Dulón, Roxana, Migración transnacional de bolivianos y bolivianas a la Argentina y su impacto en comunidades de origen, Sucre, Fundación Pasos y Grupo Chorlavi (Fondo Mink'a), 2008.

Durand, Jorge and Douglas S. Massey, «Mexican Migration to the US : A Critical Review», Latin American Research Review, vol. 27, n 2, 1992, pp. 3-43.

Faret, Laurent, "Les impacts socioculturels de la circulation migratoire dans les environnements d'origine: le cas du Mexique», in Eric Guerassimov (dir.), Migrations internationales, mobilités et développement, Paris, L'Harmattan, 2005, pp. 273-311.

Fajnzylber, Pablo and J. Humberto López, Close to Home: The Development Impact of Remittances in Latin America, Conference Edition, Washington, DC, The World Bank, 2007.

Fischer Peter A., Reiner Martin and Thomas Straubhaar, «Interdependencies between Development and Migration», in Thomas Hammar, Grete Brochmann, Kristof Tamas and Thomas Faist (eds.), International Migration, Immobility and Development, Oxford, Berg, 1997, pp. 91-132.

Fernández de Castro, Rafael, Rodolfo Garcia Zamora y Ana Vila Freyer (coord.), El programa 3x1 para migrantes. ¿ Primera política transnacional en Mexico?, México, Universidad Autónoma de Zacatecas, 2006.

Garcia Zamora, Rodolfo, Migración, remesas y desarrollo, los retos de las organizaciones migrantes mexicanas en Estados Unidos, México, Universidad Autónoma de Zacatecas, 2005.

Gosh, Bimal, Migrants' Remittances and Development: Myths, Rhetoric and Realities, Geneva, International Organization for Migration (IOM), 2006.

Hamman, Volker, «Diferencias intraregionales en la zona de alta migración zacatecana», in Raúl Delgado Wise y Beatrice Knerr (coord.), Contribuciones al análisis de la migración internacional y el desarrollo regional en México, Universidad Autónoma de Zacatecas, 2005, pp. 193-206.

Hamann, Volker, The Impact of International Labor Migration on Regional Development: The Example of Zacatecas, Mexico; Kassel, Kassel University Press, 2006.

Hammar, Thomas, Grete Brochmann, Kristof Tamas and Thomas Faist (eds.), International Migration, Immobility and Development, Oxford, Berg, 1997.

Hermele, Kenneth, «The Discourse on Migration and Development», in Thomas Hammar, Grete Brochmann, Kristof Tamas and Thomas Faist (eds.), International Migration, Immobility and Development, Oxford, Berg, 1997, pp. 133-158.

Hernandez-Coss, Raúl, Lessons from the US-Mexico Remittance Corridor on Shifting from Informal to Formal Transfer System, World Bank Working Paper, nº 47, Washington, DC, World Bank, 2005.

International Monetary Fund (IMF), «Workers' Remittances and Economic Development», chapter 2, World Economic Outlook: Globalisation and External Imbalances, Washington, DC, IMF, 2005, $<\mathrm{http}: / /$ www.imf.org/external>.

Instituto Nacional de Estadisticas, Geografia e Informatica (INEGI), Encuesta Nacional de Ingresos y Gastos de Hogares, Mexico, 2000.

Inter-American Development Bank (IDB), Enviando Dinero a Casa: Marcador de la Industria de Remesas, Washington, DC, IDB 2006.

Inter-American Development Bank (IDB), Remittance Flows to Latin America and the Caribbean, 2004, Washington, DC, IDB, 2004.

Inter-American Development Bank (IDB), Remittance Senders and Receivers: Tracking the Transnational Channels, Washington, DC, IDB, 2003.

Inter-American Development Bank (IDB), Remittances 2005: Promoting Financial Democracy, Washington, DC, IDB, 2006.

Inter-American Development Bank (IDB), Sending Money Home: Remittance Recipients in the Dominican Republic and Remittance Senders from the United States, New York, IDB and Columbia University, 2004.

Inter-American Development Bank (IDB), State by State Survey of Remittance Senders : United States to Latin America, Washington, DC, IDB, 2004.

Inter-American Development Bank (IDB), Survey of Remittance Senders: United States to Latin America, Washington, DC, IDB, 2002.

Inter-American Dialogue (IAD), Making the Most of Family Remittances, Second Report of the InterAmerican Dialogue Task Force on Remittances, Washington, DC, IAD, 2007.

Knerr, Beatrice, «Dinàmicas económicas regionales frente a la migración laboral internacional. Teorías y experiencias globales», in Raúl Delgado Wise y Beatrice Knerr (coord.), Contribuciones al análisis de la migración internacional y el desarrollo regional en México, Universidad Autónoma de Zacatecas, 2005, pp. 137-170.

Loaiza, Alejandrino S., L. Herrea y César Sotomayor, Las Remesas de los Migrantes Quillabambinos en Italia y sus Implicancias en el Desarrollo Local, Cusco, Proyecto de Desarrollo Corredor Puno-Cusco y Grupo Chorlavi, 2008. 
Lozano Ascencio, Fernando, «De excluidos sociales a heroes sexenales. Discurso oficial y remesas en Mexico», in Raúl Delgado Wise y Beatrice Knerr (coord.), Contribuciones al análisis de la migración internacional y el desarrollo regional en México, Universidad Autónoma de Zacatecas, 2005, pp. 41-65.

Neira Orjuela, Fernando, Sistematización de las experiencias en el uso productivo de remesas para el fomento del desarrollo: un estudio de caso en proyectos productivos del municipio de Atacheo de Regalado, en el Estado de Michoacán de Ocampo, México, México, UNAM y Grupo Chorlavi (Fondi Mink'a), 2008.

Organisation de coopération et de développement économiques (OCDE), Migration et développement, un nouveau partenariat pour la coopération, compte rendu de conférence, Paris, OCDE, 2008.

Orozco, Manuel, Transnational Families: Lives on the Edge, but in Pursuit of Change, 2006, cité par Ezra Rosser, Remittances, College of Law Research Paper, $n^{\circ}$ 40, Washington, DC, American University College of Law, 2008, <http://ssrn.com/abstract=1024177>.

Osorio Perez, Flor, W. Mejia, G. Restrepo, A. Milena y Y. Cifuentes, De Productor de Café a Pueblo Emigrante, Bogotà, Universidad Javeriana, Universidad tecnologica de Pereira y Grupo Chorlavi (Fondo Mink'a), 2008.

Petree, Jennifer y Tahira Vargas, Dominicanos en Suiza. Pautas, prácticas e impactos de la migración transnacional y el envió de remesas que vinculan Republica Dominicana y Suiza, EPFL, Cahiers du LaSUR, no 8, Lausanne, Ecole polytechnique fédérale de Lausanne, 2005.

Rosser, Ezra, Remittances, College of Law Research Paper, ${ }^{\circ}$ 40, Washington, DC, American University College of Law, 2008, <http://ssrn.com/abstract=1024177>.

Straffon, Beatriz, Paola Lopez y Anally Castellanos, «El Programa 3x1 para Migrantes en Oaxaca. Migracion indigena, practicas comunitarias y costumbres de participacion », in Rafael Fernández de Castro, Rodolfo Garcia Zamora y Ana Vila Freyer (coord.), El programa 3x1 para migrantes. ¿ Primera política transnacional en Mexico?, México, Universidad Autónoma de Zacatecas, 2006, pp. 197-223.

Suki, Leonora, Financial Institutions and the Remittance Market in the Domican Republic, New York, Center on Globalization and Sustainable Development, The Earth Institute at Columbia University, 2004.

Western Union, Western Union Expands Initiative to Promote Community Economic Development in Mexico; Mexican State of Michoacan the Second State to benefit from 4x1 Program, Press Release, 23 June 2006, <http://ir.westernunion.com/investor/releasedetail.cfm?ReleaseID=219081>.

Wilson, John F., Progress Report on CEMLA Remittances Compilation Manual for LAC Countries, available on Internet page «Remittances Statistics: First Meeting of the Luxemburg Group June 2006», $<$ http://www.imf.org/external/np/sta/bop/2006/luxgrp/060106.htm>.

World Bank, Global Economic Prospects 2006: Economic Implications of Remittances and Migration, Press launch, with Christopher Neal, François Bourguignon, Hans Timmer, Dilip Ratha, William Shaw, Washington DC, The World Bank, 16 November 2005.

World Bank, Global Economic Prospects 2006: Economic Implications of Remittances and Migration, Washington, DC, World Bank, 2006.

World Bank, The Development Impact of Workers' Remittances in Latin America, Report, $\mathrm{n}^{\circ} 37026$, Washington, DC, World Bank, 2006.

Wucker, Michele, «Remittances, the Perpetual Migration Machine», World Policy Journal, vol. 21, nº 2 , Summer 2004.

Yépez del Castillo, Isabel et Gioconda Herrera Mosquera (dir.), Nouvelles migrations latino-américaines en Europe, Barcelona, Publicacions i Edicions de la Universitat de Barcelona, 2008. 